10. STELLAR MODELS VERSUS OBSERVATIONS 


\title{
THE EVOLUTION OF MASSIVE STARS
}

\author{
A. MAEDER \\ Geneva Observatory, CH-1290 Sauverny, Switzerland
}

\section{Introduction}

Massive stars are the crossroads of many important astrophysical problems and thus a proper understanding of their evolution is very needed. They are the main sources of UV radiation, by heating the interstellar dust they produce the far-IR luminosities of galaxies. They are the precursors of Supernovae and also the main sources of nucleosynthesis. They are visible in distant galaxies and the recent observations of starbursts have shown their major role in the spectral and chemical evolution of galaxies. They begin to be observed in the galactic center and in regions of star formation around galactic nuclei.

The properties of massive stars have been extensively studied over the last decade (cf. Chiosi \& Maeder, 1986; Maeder \& Conti, 1994). In Geneva (for example !), the following grids have been made:

- Pre-MS evolution with accretion (Bernasconi \& Maeder, 1996; Bernasconi, 1996)

- MS and post-MS evolution in the range of 0.8 to $120 \mathrm{M}_{\odot}$ for:

\begin{tabular}{llll}
\hline $\mathrm{Z}=0.10$ & $\mathrm{Y}=0.48$ & Mowlavi et al., 1997 & (paper VIII) \\
$\mathrm{Z}=0.04$ & $\mathrm{Y}=0.34$ & Schaerer et al., 1993b & (paper IV) \\
$\mathrm{Z}=0.02$ & $\mathrm{Y}=0.30$ & Schaller et al., 1992 & (paper I) \\
$\mathrm{Z}=0.008$ & $\mathrm{Y}=0.264$ & Schaerer et al., 1993a & (paper II) \\
$\mathrm{Z}=0.004$ & $\mathrm{Y}=0.252$ & Charbonnel et al., 1993 & (paper III) \\
$\mathrm{Z}=0.001$ & $\mathrm{Y}=0.243$ & Schaller et al., 1992 & (paper I) \\
\hline
\end{tabular}

- Models of stars with $\mathrm{M}>15 \mathrm{M}_{\odot}$ for the above $(\mathrm{Z}, \mathrm{Y})$ values have been calculated with mass loss rates increased by a factor of 2 (Meynet et

T.R. Bedding et al. (eds.),

Fundamental Stellar Properties: The Interaction between Observation and Theory, 313-322.

(c) 1997 IAU. Printed in the Netherlands. 
$a l .$, 1994, paper v).

- Models for Horizontal Branch (HB), post-HB and AGB stars have been constructed for various (Z,Y), (cf. Charbonnel et al., 1996, paper VI).

- Models for low mass stars in the range 0.4 to $1 \mathrm{M}_{\odot}$ for $\mathrm{Z}=0.02$ and $\mathrm{Z}=0.001$ have been made (cf. Charbonnel et al., 1997, paper VII).

- Complete tables of isochrones for the various $\mathrm{Z}$ and for ages from $2 \times$ $10^{6} \mathrm{yr}$ to $2 \times 10^{10} \mathrm{yr}$.

- A code for calculating isochrones with various color indices, synthetic open clusters, population synthesis is kindly provided on request.

These data are available at the CDS in Strasbourg or by e-mail on request (georges.meynet@obs.unige.ch).

\section{Pre-MS evolution}

New pre-MS models have been made (cf. Bernasconi \& Maeder, 1996; Bernasconi, 1996). They follow the accretion scenario firstly proposed by Palla and Stahler (1991, 1992, 1993; see also Beech \& Mitalas, 1994). The basic idea is the following one. Molecular clouds can become gravitationally unstable and produce a large inflow of matter, which will be accreted in the associated star formation process. In the central volume, matter organizes itself in a spatially thin and very extended disk (several $10^{2} \mathrm{AU}$ ) from which matter is progressively accreted onto the central protostellar core. The pre-MS evolution is very different according to stellar mass. For a $1 \mathrm{M}_{\odot}$ star the accretion period is terminated at sufficiently early stages, so that the Hayashi phase occurs like in models without accretion and the pre-MS stage lasts the classical $3 \times 10^{7} \mathrm{yr}$ (Palla \& Stahler, 1993). For stellar masses larger than $7-10 \mathrm{M}_{\odot}$, the accretion time becomes longer than the Kelvin-Helmholtz time-scale, and massive stars continue to accrete matter hidden within their molecular cloud, while they are already centrally contracted and enter their hydrogen burning phase.

A basic parameter is the mass accretion rate. In our models, the equilibrium equation of cloud models are solved with the assumption that the clouds have both a thermal pressure and a non-thermal contribution supporting them. The non-thermal effect is based on the velocity dispersionsize relation by Larson (1981). This way, accretion rates are obtained; they are growing function of the already accreted stellar mass.

Fig. 1a shows two birthlines in the accretion scenario. The birthline is the path followed by a continuously accreting star. Depending on the moment when the accretion becomes negligible, the star will stop its rising evolution on the birthline. Various mass ranges on the birthline of a massive star can be distinguished (Bernasconi \& Maeder, 1996). 

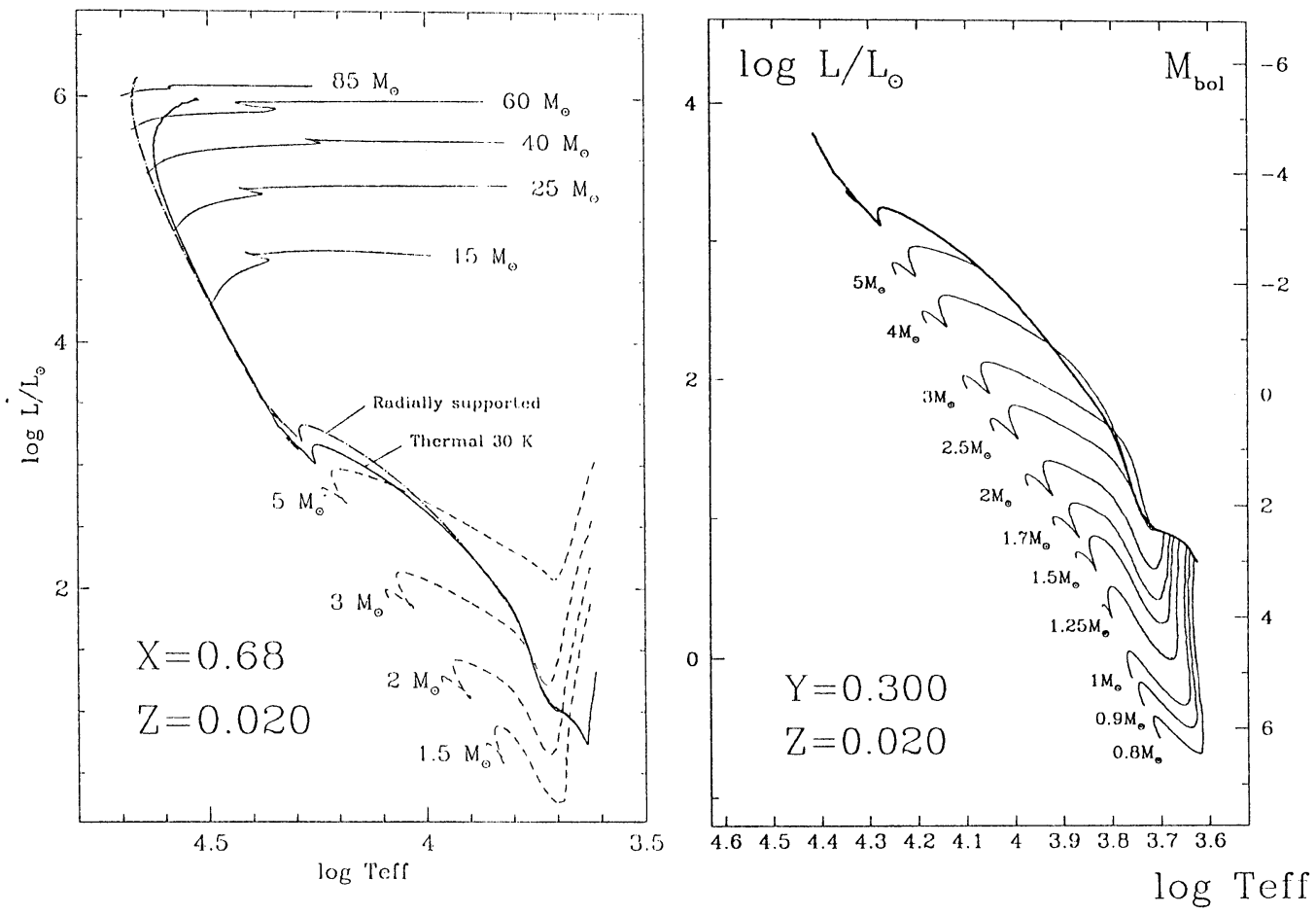

Fig.1a and 1b. Pre-MS evolutionary tracks with accretion. Fig. 1a: Standard pre-MS tracks are given without accretion (broken line); the continuous lines for 15 to $85 \mathrm{M}_{\odot}$ are post-MS tracks. The two upwards going lines are birthlines for two different hypotheses on the cloud support. Fig. 1b: Detailed pre-MS tracks with accretion, the thicker line is the birth line, which is followed by massive pre-MS stars (cf. Bernasconi \& Maeder, 1996; Bernasconi, 1996).

In particular, at about $8 \mathrm{M}_{\odot}$, the star reaches its minimum radius, which we define as the point where the star reaches the ZAMS. This very moment is preceded by the slow ignition of $\mathrm{CN}$ burning and by some ${ }^{3} \mathrm{He}$ spikes due to the successive back and forth motions of the convective core. Fig. 1b shows the details of pre-MS tracks leaving the birth line, which is the path followed by massive pre-MS stars.

These new models show several interesting features:

- The pre-MS lifetimes for massive stars with accretion are much larger (i.e. about $1.3 \times 10^{6} \mathrm{y}$ for $25 \mathrm{M}_{\odot}$ ) than for standard evolution (i.e. $7.4 \times$ $10^{4} \mathrm{yr}$ for a $25 \mathrm{M}_{\odot}$ star).

- At the time it becomes visible, a newly formed star with $M \geq 40 M_{\odot}$ has already burnt some of its central hydrogen and consequently has already 
moved away from the formal "zero age sequence". This may explain the lack of $\mathrm{O}$-type stars close to the formal zero age sequence.

- The initial conditions for post-MS models are different; in particular the core sizes are 5-10\% smaller. The remaining MS lifetimes are reduced.

- As the most massive stars spend a substantial fraction of their total life hidden in their parental molecular cloud, their true number is underestimated and the slope of the IMF is somehow flatter than usually considered.

\section{Summary of mass loss effects in evolution. Observations}

Mass loss is a dominant effect of massive star evolution. We can even say that massive stars nearly fully evaporate during their evolution. The very low final masses in the range $5-10 \mathrm{M}_{\odot}$ for solar metallicity are very well confirmed by the luminosity distribution of WR stars (Maeder \& Meynet, 1994).

A basic assumption in models of different metallicity $\mathrm{Z}$ is the dependence of the mass loss rates $\dot{\mathrm{M}}$ on $\mathrm{Z}$. We assume a relation of the form $\dot{\mathrm{M}} \sim \mathrm{Z}^{\alpha}$, with $\alpha \simeq 0.5$ (cf. Kudritzki et al., 1991). The value of $\alpha$ for supergiants is still a major uncertainty.

\subsection{O-STARS:}

During MS evolution, the effects of mass loss at the observed rates are rather small:

- The sizes of the convective core are reduced and thus their luminosities as well.

- However, the core mass fractions are larger than in a star of the same actual mass, but evolving at constant mass. The stars evolving with mass loss are also over-luminous with respect to their actual masses.

- The MS lifetimes undergo little increases $(<10 \%)$.

- A certain MS widening occurs around $60 \mathrm{M}_{\odot}$ for solar composition and at lower masses for higher Z (cf. Fig. 2).

- Mass loss at the observed rate is unable to produce an increase of the $\mathrm{N} / \mathrm{C}$ and N/O ratios during the MS for stellar masses below about $70 \mathrm{M}_{\odot}$.

Concerning the observations, the most interesting result is that by Herrero et al. (1992), who shows that fast rotating $\mathrm{O}$-stars exhibit $\mathrm{He}-$ enhancements. This observations, joined to the existence of ON-stars, suggest that at least a fraction of $\mathrm{O}$-stars is experiencing additional mixing processes, either due 


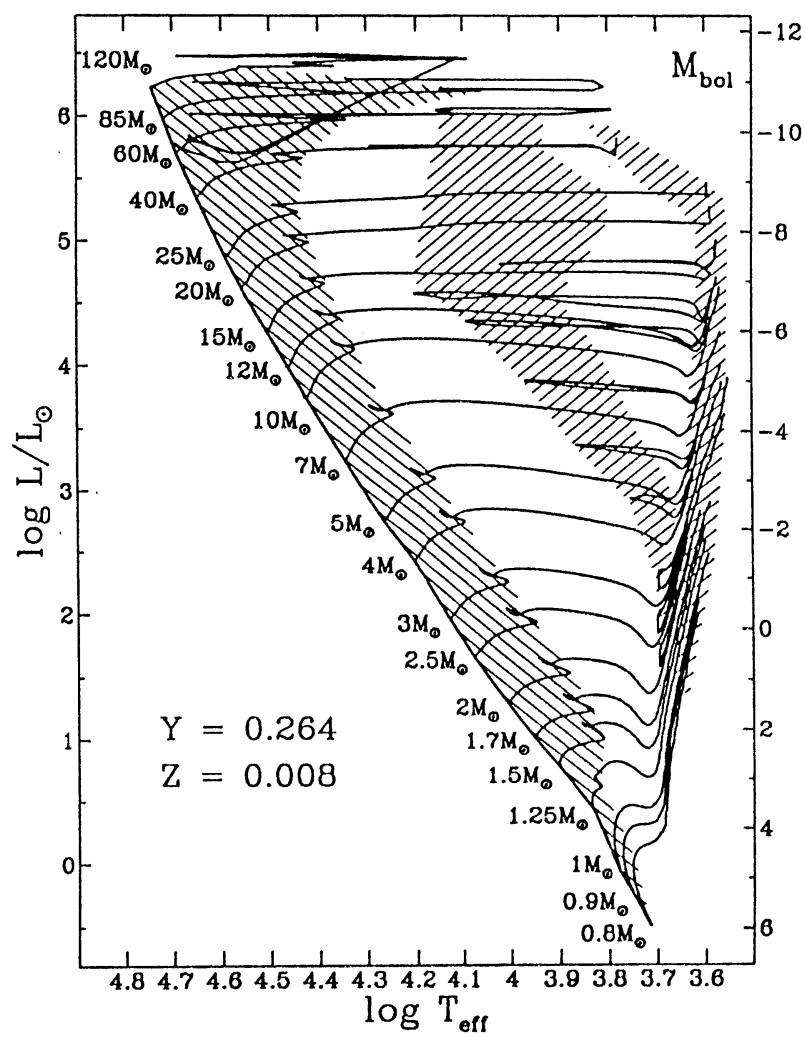

Figure 2. Evolutionary tracks appropriate to LMC, composition with indications of the slow phases of nuclear burning.

to rotation and/or to tidal mixing in binaries. Another point, possibly related to the previous one, is the fact that the upper MS of young clusters is more elongated than predicted (cf. Meynet et al., 1993). This could be the cause of the absence of a visible gap at the end of the MS.

\subsection{SUPERGIANTS, THE B/R RATIO:}

At the present stage, the problems of blue, yellow and red supergiants are more intricate. The models, on the whole, correctly predict the observed ratios of red supergiants to MS stars for galactic clusters in the range of $3 \times 10^{6} \mathrm{yr}$, (cf. Meynet, 1993). However, the study of clusters in the SMC, LMC and Galaxy show that the number ratio B/R of blue-to-red supergiants is an increasing function of metallicity (see Langer \& Maeder, 1995 for recent ref.). Clusters in inner galactic regions have much larger $B / R$ ratios than in the SMC, where large numbers of red supergiants are present as in NGC 330 . Up to now, no sets of models are able to correctly 
reproduce the observed trend of B/R with $\mathrm{Z}$ (cf. Langer \& Maeder, 1995). Some models give the correct B/R at solar Z (models with Schwarzschild criterium), but fail generally at low $\mathrm{Z}$. At the opposite, models with the Ledoux criterion give the right $B / R$ at low $\mathrm{Z}(\mathrm{Z}=0.003)$, but fail generally at solar Z. Thus, the problem may have to do with mixing, in addition to mass loss.

Mass loss also strongly affects the $\mathrm{B} / \mathrm{R}$ ratios. If there is no mass loss (and Schwarzschild's criterion), there are no red supergiants; for growing $\dot{\mathrm{M}}$-rates, red supergiants appear, thus B/R decreases. For large enough $\dot{\mathrm{M}}-$ rates, red supergiants themselves disappear, quickly turning to WR stars. In this regime, one has a growing number of red supergiants for lower $\mathrm{Z}$, as observed. The problem is that this situation occurs for too large $\dot{M}$-rates compared to the observations.

The physical reason for the presence or absence of red supergiants is that due to mass loss, the fractional mass of the He core becomes higher and also produces an outward shifting of the H-burning shell. This tendency favors redwards evolution up to some critical value of the core mass fraction $\mathrm{q}_{\mathrm{c}}$ and bluewards after $\left(\mathrm{q}_{\mathrm{c}}=0.67,0.77,0.97\right.$ at 60,30 and $15 \mathrm{M}_{\odot}$ respectively). Interestingly enough, the presence of some mild mixing in the star will contribute to the growth of the actual core mass fraction, thus the critical $\mathrm{q}_{\mathrm{c}}$ will be reached more easily. Also, mixing will influence the location and importance of the $\mathrm{H}$-burning shell, and the response of the star is then more complex with in particular the presence or absence of blue loops (cf. Langer, 1991). Models in progress with rotational mixing may help to solve this longstanding problem. This possibility is also supported by the evidences of $\mathrm{N} / \mathrm{C}$ and He enhancements in blue and yellow supergiants (Venn, 1995), which are not predicted for non rotating models.

Very often, star models in the supergiant stage are close to a neutral equilibrium between a blue and red location. Processes affecting the envelope structure may give the final kick to the blue or the red. Such uncertainties are of course a difficulty to interprete the populations of blue and red supergiants, particularly at low metallicities. However, these uncertainties have very little consequences on the subsequent evolution, since further mass loss is quickly removing the outer envelopes, with or without additional mixing, to let finally bare cores identified as Wolf-Rayet stars. The evolution of WR stars are dominated by mass loss and the effects of previous differences are washed out. 


\subsection{WOLF-RAYET STARS}

WR stars have an average mass below $10 \mathrm{M}_{\odot}$, they are over-luminous with respect to main-sequence stars. They fit well the mass-luminosity relation of bare-core models (Smith \& Maeder, 1989). More generally, bare cores containing no hydrogen (roughly corresponding to WNE and WC stars) fit a $\mathrm{M}-\mathrm{L}-\dot{\mathrm{M}}-\mathrm{R}-\mathrm{T}_{\text {eff }}$ - chemistry relation (cf. Schaerer \& Maeder, 1992). To get $\mathrm{R}$ and $\mathrm{T}_{\text {eff }}$, a proper account for the optically thick wind has to be made. A simple procedure has been used in Schaller et al., (1992) and by Schaerer and Maeder; despite its simplicity it is consistent with the results of the complete stellar models, including the interior, atmosphere and winds (cf. Schaerer et al., 1996).

The mass loss rates in the WR stages without hydrogen seem to have little or no dependence on metallicity. The main parameter is the actual mass of the WR stars, and a relation of the form $\dot{M}_{\mathrm{WR}} \sim M_{\mathrm{WR}}^{\alpha}$ with a value $\alpha \approx 2$ has been proposed by Langer (1989), while Moffat (1995) suggests a rather linear dependence.

At present, the following filiations can be distinguished for massive stars (cf. Chiosi \& Maeder, 1986; Crowther et al., 1995; Langer et al., 1994).

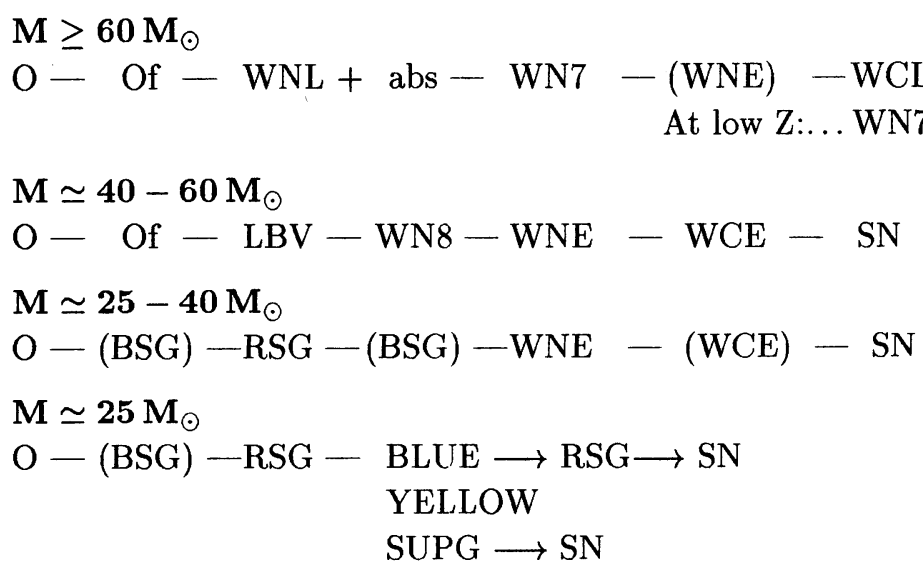

Several uncertainties remain, e.g. whether stars with $M \geq 60 M_{\odot}$ avoid the LBV (Luminous Blue Variables), or whether the LBV turns into WN8 as supposed by Crowther et al. Differences in the mass limits and in the exact sequences may also depend on $\mathrm{Z}$. For example, for $Z \geq 0.02$, we likely have $\mathrm{WNL} \rightarrow \mathrm{WCL}$, avoiding the WNE stage. For $\mathrm{Z}<0.02, \mathrm{WNL} \rightarrow \mathrm{WCE} \rightarrow$ WO, mostly avoiding the WCL stage, and WNE do not lead to WC stars.

In WN stars, CNO ratios are in agreement with equilibrium values, thus CNO abundances are a test for nuclear cross-sections rather than for model assumptions. Surface helium contents range from $\mathrm{Y}_{s}=0.4$ (gen. in WNL) 


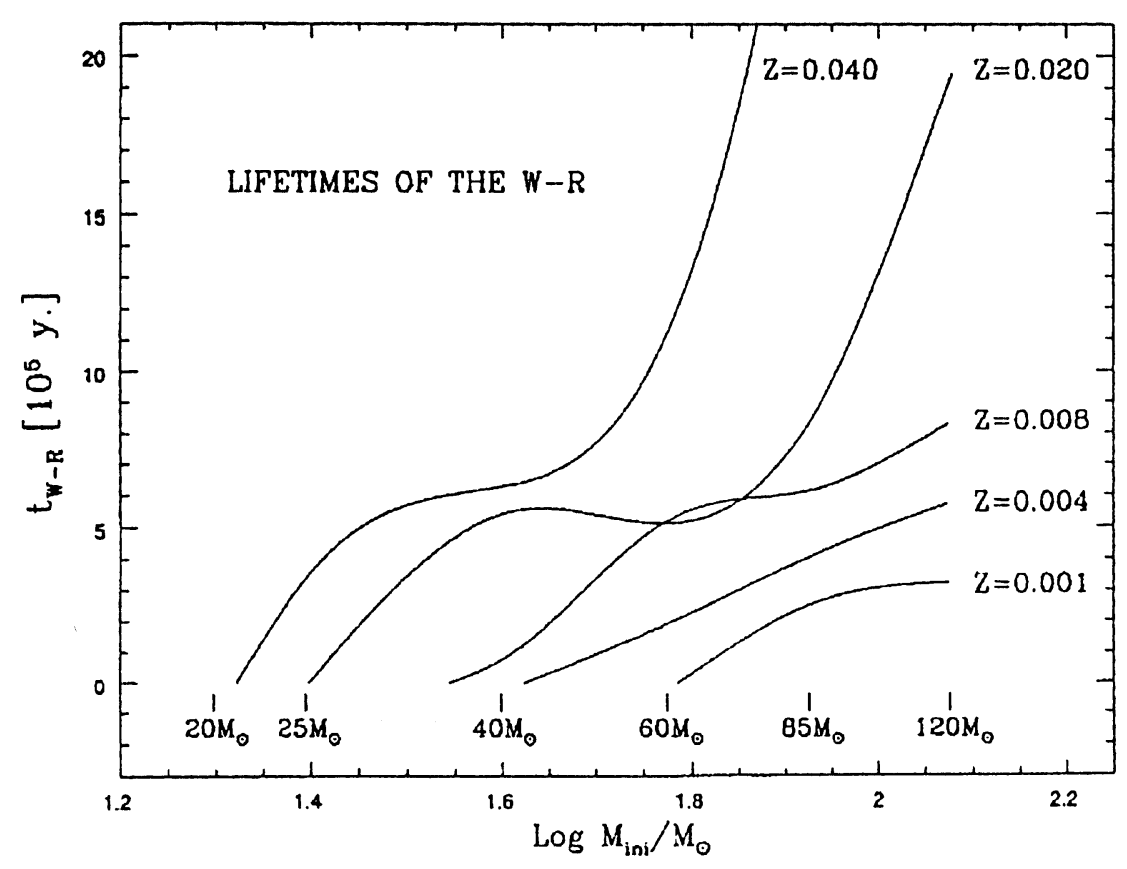

Figure 3. WR lifetimes as a function of $\mathrm{M}$ and $\mathrm{Z}$.

to $\mathrm{Y}_{s}=0$ (gen. in WNE). The $\mathrm{Y}_{s}$ vs. $\log \mathrm{L}$ diagram is a key diagram for WN stars (cf. Langer et al., 1994; Maeder 1995). In this diagram it seems, however, difficult to account for the WNE stars of low luminosity with non-zero $\mathrm{Y}_{s}$. This might be a signature of mixing.

For WC stars, the sequence WC9, WC $8 \ldots$ WC4, W0 seems to be a sequence of increasing (C+O)/He (cf. Smith \& Hummer, 1988; Smith \& Maeder, 1991). This ratio is preferable to $\mathrm{C} / \mathrm{He}$, which does not vary monotonously during evolution. WC abundances are basically a test of model assumptions, since they are products of partial He-burning. The models made at various $\mathrm{Z}$ allow to understand the main observed properties of WC stars in galaxies (cf. Smith \& Maeder, 1991): 1) WCL stars are preferentially created at high $\mathrm{Z}, 2$ ) at a given $\mathrm{Z}$, WCL stars are brighter than WCE stars, 3) stars of a given WC subtype are brighter at low $\mathrm{Z}$.

The number ratios $\mathrm{WR} / \mathrm{O}$ and $\mathrm{WC} / \mathrm{WN}$ change very much according to galaxies and locations in galaxies (cf. Maeder \& Conti, 1994). To carefully discuss this important property, we must distinguish: 
a) galaxies and areas in galaxies, where the assumption of constant star formation rate (SFR) over the last $10^{7} \mathrm{yr}$ is valid and

b) starburst regions and starburst galaxies, where the SFR has greatly varied in recent times.

For case a), the main effect responsible for changes in massive star population is metallicity Z. Fig. 3 shows the lifetimes of WR stars as a function of $\mathrm{M}$ and $\mathrm{Z}$ for models with enhanced $\dot{M}$-rates (cf. Maeder \& Meynet, 1994). When integrated over the current mass spectrum and with the hypothesis of constant SFR, these lifetimes well reproduce the observed number ratios WR/O in nearby galaxies (cf. also Maeder \& Conti, 1994).

It does not seem necessary to advocate the contribution of mass transfer in many binaries. Case b) of starbursts is quite different. There it appears that the observations pick up these objects at a specific time after a very short starburst, so that they show very high $\mathrm{WR} / \mathrm{O}$ ratios. Theses ratios may be up to an order of magnitude larger than in case a). The situation has been discussed by Arnault et al. (1989), Maeder \& Conti (1994) and by Meynet (1995), who has made very complete calculations.

The WR stars are most useful objects, even observable in the integrated spectrum of galaxies; thus WR stars may give us an access to the properties (age, SFR, mass spectrum etc.) of distant starbursts.

\section{References}

Arnault, P., Schild, H. (1989) $A \& A$ 224, 73

Beech, M., Mitalas, R. (1994) ApJS 95, 517

Bernasconi, P. A. (1996) A $\& A$ 120, 57

Bernasconi, P., Maeder, A. (1996) $A \& \&$ 307, 829

Charbonnel, C., Meynet, G., Maeder, A., Schaller, G., Schaerer, D. (1993) A\&AS 101, 415 (paper III)

Charbonnel, C., Meynet, G., Maeder, A., Schaerer, D. (1996) $A \& A S$ 115, 339 (paper VI)

Charbonnel, C., D=E4ppen, W., Bernasconi, P., Maeder, A., Meynet, G., Schaerer, D., Mowlavi, N. (1997) $A \boxminus A S$, in press (paper VII)

Chiosi, C., Maeder, A. (1986) $A \& A$ 24, 329

Crowther, P. A., Smith, L. J., Hillier, D. J., Schmutz, W. (1995) $A \& A$ 293, 427

Herrero,, A., Kudritzki, R. P., Vichez, J. M., Kunzer, D., Butler, K. (1992) $A \xi A$ 261, 209

Kudritzki, R. P., Pauldrack, A., Puls, J., Voels, S. R.. (1991), IAU Symp. 148,

Ed. R. Haynes and D. Milne, p. 279

Langer, N. (1989) $A \& A$ 220, 135

Langer, N. (1991) $A \& A$ 252, 669

Langer, N., Hamann, W. R., Lennon, M., Najarro, F., Pauldrach, A. W. A., Puls, J. (1994) $A \& A$ 290, 819

Langer, N., Maeder, A. (1995) $A \& A$ 295, 685

Larson, R. B. (1981) MNRAS 194, 809

Maeder, A. (1995) in "Wolf-Rayet stars: Binaries Colliding Winds, Evolution" IAU Sump. 163, Eds. K. van der Hucht and P.M. Williams p. 280

Maeder A., Conti, P. (1994) ARAA 32, 227

Maeder A., Meynet, G. (1994) $A \mathscr{E} A$ 287, 803 
Meynet, G. (1993) in "The Feedback of Chemical Evolution on the Stellar Content of Galaxies", Eds. D. Alloin, G. Stasinska, Obs. de Paris, p. 40

Meynet, G. (1995) $A \& A$ 298, 767

Meynet, G., Maeder, A., Schaller, G., Schaerer, D., Charbonnel, C. (1994) A\&AS 103, 97 (paper v)

Meynet, G., Mermilliod, J.-C., Maeder, A. (1993) A\& $A S$ 98, 477

Moffat, A. (1995) in "Wolf-Rayet stars: Binaries Colliding Winds, Evolution" IAU Symp. 163, Eds. K. van der Hucht and P.M. Williams p. 213

Movlawi, N., Schaerer, D., Meynet, G., Bernasconi, P.A., Charbonnel, C., Maeder, A. (1997) $A \& A S$, in press (paper VIII)

Palla, F., Stahler, S. W. (1991) ApJ 375, 288

Palla, F., Stahler, S. W. (1992) ApJ 392, 667

Palla, F., Stahler, S. W. (1993) ApJ 418, 414

Schaerer D., Maeder, A. (1992) $A \mathscr{6} A$ 263, 129

Schaerer, D., Meynet, G., Maeder, A., Schaller, G. (1993a) $A \& A S$ 98, 523 (paper II)

Schaerer, D., Charbonnel, C., Meynet, G., Maeder, A., Schaller, G. (1993b) $A \& A S$ 102, 339 (paper IV)

Schaerer, D., de Koter, A., Schmutz, W., Maeder, A. (1996) $A \& A$ 312, 475

Schaller, D., Schaerer, D., Meynet, G., Maeder, A. (1992) $A \& A$ 96, 269 (paper I)

Smith, L., Hummer, D.G. (1988) MNRAS 230, 511

Smith, L., Maeder, A. (1989) $A \mathscr{B} A$ 211, 71

Smith, L., Maeder A. (1991) $A \& A$ 241, 77

Venn, K. (1995) ApJS 99, 659

\section{DISCUSSION}

BENGT GUSTAFSSON: I was impressed with your good agreement between the predicted number of Wolf Rayet Stars relative to the number of 0 stars for different metallicities and the observed ratio for different galaxies. Are there any free parameters in that fit?

ANDRE MAEDER: The good agreement between the observed and theoretical number ratios suggests that the fraction of WR owing their existence to binary mass transfer is small. This is certainly one free parameter in the comparisons.

STEVE KAWALER: How many free parameters are used in your formulations for rotational mixing?

ANDRE MAEDER: We have been working over the last two years on the Richardson criterion, meridional circulation, thermal instabilities, etc., in order to have a theory as consistent as possible. I believe there is no interest nowadays to build 'parametrized' theories.

JOHN NORRIS: Is the nitrogen produced by your rotating models primary or secondary?

ANDRE MAEDER: The nitrogen enrichment we are getting is secondary during the 0-type star and super-giants phases. However, it is quite possible that rotational mixing may produce some primary nitrogen during the WR phases, when some freshly produced ${ }^{12} \mathrm{C}$ is diffused in H-burning regions. We must check that quantitatively. 